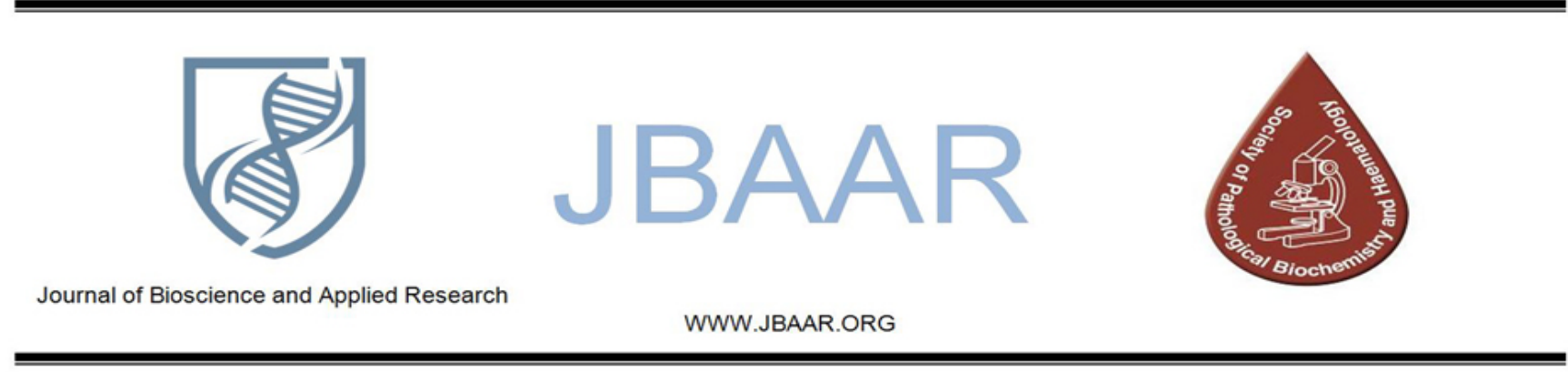

\title{
Intestinal Form of Rabbit Haemorrhagic Disease in Growing Rabbits
}

\section{(Oryctolagus cuniculus)}

\author{
Abou-Shafey A. E* ${ }^{1}$; Metwally A. $\mathrm{Y}^{2}$; Massoud. A. A ${ }^{1}$; Barakat M. E . and Elwan M. $\mathrm{M}^{1}$ \\ ${ }^{1}$ Zoology Department, Faculty of Science, Tanta University. \\ ${ }^{2}$ Department of poultry and rabbit disease Animal Health Research Institute, Kafr-El-Sheikh. \\ ${ }^{3}$ Department of biochemistry Animal Health Research Institute, Kafr-El-Sheikh. \\ (Corresponding author e.mail ahlam.aboushafie@science.tanta.edu.eg)
}

\begin{abstract}
Rabbit Haemorrhagic Disease (RHD) is extremely acute highly fatal, contagious disease with mortality rates of $80-90 \%$ of the infected rabbits. RHD causes hepatic, intestinal and lymphoid necrosis with massive terminal intravascular coagulopathy. The etiological agent is a member of caliciviridae lagovirus, Rabbit Haemorrhagic Disease Virus (RHDV); it is a single stranded RNA, nonenveloped and replicates in the cytoplasm. In pathogenesis studies, the primary sites of replication were in the small intestinal crypt and villous epithelium, hepatocytes and splenic lymphocytes. Apoptosis (programmed cell death) has been reported as being a constant feature of the pathogenesis of RHD. This work was planned to study the lesions associated with RHDV in small intestine at different intervals. Eighteen growing New Zealand rabbits (Oryctolagus cuniculus) aged 2-3 months allotted into two equal groups: control group (non infected) and infected group in which rabbits were experimentally inoculated with rabbit hemorrhagic disease virus (RHDV) through the nostril. All animals were dissected at 24, 48 and 72 hrs post infection. Histopathological, histochemical, immunohistochemical and biochemical studies were done for small intestine.

Macroscopic lesions in infected grower rabbits were consistent with RHD infection including congestion and haemorrhages of lung, liver necrosis and splenomegaly. Moreover, congestion of small intestine with multiple focal necrotic spots appeared from serosa and mucosa of intestine. Histopathological findings of the small intestine 24 hrs post infection (pi) showing necrosis of the crypts
\end{abstract}

and villi atrophy, at 48 hrs pi shortening of villi and severe lymphocytic infiltration of the lamina propria were seen. 72 hrs pi showing severe atrophy and destruction of both villi and crypts. Immunohistochemical labeling for RHDV antigen on small intestine at different intervals 24, 48 and 72 hrs pi showed that epithelial cells and areas of focal necrosis exhibit strong immunolabeling in the intestinal villi where reactivity increases progressively. Serum biochemistry revealed highly significant increase in AST, ALT, urea and creatinine. To the best of our knowledge, it is the first report of the macroscopic lesions of small intestine in RHDV infected rabbits.

Keywords: Rabbit Haemorrhagic Disease, Rabbit Haemorrhagic Disease Virus, Rabbits, Small Intestine, Pathogenesis, Immunohistochemistry, Transaminases

\section{Introduction}

Rabbit hemorrhagic disease (RHD) is an acute viral disease caused by a calicivirus (Parra and Prieto, 1990) that was first described in China (Liu et al. 1984) and since then, it has been causing worldwide epidemic fatal outbreaks. RHD is per-acute viral of rabbits (Oryctolagus cuniculus) causing hepatic, intestinal and lymphoid necrosis and massive terminal intravascular coagulation (Oie, 2015). The causative agent of the disease is Rabbit Haemorrhagic Disease Virus (RHDV) that has a $40 \mathrm{~nm}$ icosahedric capsid with only one major structural protein of about $60 \mathrm{kDa}$ (VP60). The virion contains a full-length genomic positive RNA, and a subgenomic RNA (Meyers et al. 1991a and Meyers et al. 1991b). 
Gregg et al. (1991) reported that infected rabbits with the virus die suddenly, following 6 to 24 hours fever accompanied by mild clinical signs; most rabbits appear depressed, some show a variety of neurologic signs including excitement, incoordination, opisthotonos and paddling, sometimes rabbits emit a terminal squeal, few have a terminal sero-sanguinous nasal discharge as a result of acute pulmonary oedema and congestion. Gross pathologic lesions include congestion and haemorrhages in the lungs, a fine lobular pattern of necrosis in the liver, resulting in a pale liver with a distinct lobular pattern, and splenomegaly. They added that haemorrhages and congestion may be seen in many other organs and are due to a terminal intravascular coagulopathy rather than to primary involvement of those organs. Another characteristic lesion is necrosis of the small intestinal crypts, subsequent villous atrophy and sloughing of infected villous epithelial cells into the intestinal lumen, that may vary in severity and is often a segmental lesion involving only a few areas of the small intestine at a time (Gregg and House, 1989).

Programmed cell death is a constant feature in rabbits experimentally infected with RHDV, the process affected mainly hepatocytes, but also macrophages and endothelial cells presented morphologic hallmarks of apoptosis, expressing all these cell types viral antigens as determined by immunohistochemistry. The occurrence of programmed cell death was correlated with the appearance of the RHDV induced pathology in tissues by DNA fragmentation detection in situ (Alonso et al. 1998). Apoptosis of intravascular monocytes and endothelial cells was observed together with fibrin thrombi in blood vessels. Since apoptotic cells are known sites of enhanced procoagulant activity, apoptosis of these cell populations might constitute a first step in the pathogenesis of Disseminated Intravascular Coagulation (DIC) and a common pathway to other viral hemorrhagic fevers (Ueda et al. 1992).

According to Kevin et al. (2012), the immunohistochemical staining of tissues using the antiRHDV mouse monoclonal 6G2 (1:700) and the AvidinBiotin complex (Vectastain ABC Kit; Vector Laboratories Ltd., Peterborough, UK) confirmed the presence of RHDV-N11 VP1 in the liver, heart, kidney, spleen, lung, and intestine of infected rabbits, moreover immunohistochemical analyses of tissues from the subcutaneously inoculated kit that died 48 hrs post infection showed areas of focal necrosis and strong immunolabeling in the intestinal villi of the small intestine.In addition, Sheng et al. (2011) explained the exponential increases in AST and ALT that may be a strong prediction of the fulminant consequence in RHDV infection and the profound changes in serum liver enzymes, particularly AST and ALT, may be used as useful parameters in monitoring the progression of RHDV infection. This work was planned to study the lesions associated with RHDV in small intestine at different intervals.

\section{Material and Methods}

\section{1- Rabbits and Experimental Design:}

Eighteen New Zealand healthy rabbits (Oryctolagus cuniculus) aged 2-3 months were used in this study. Rabbits were purchased from private farm and maintained at regular light and dark cycles, and provided with food and water ad libitum. Random blood samples were collected from the ear vein from all rabbits for preparation of serum samples before the experimental infection, Haemagglutination Inhibition test (HI) were done to ensure they were free from RHDV antibodies (RHD seronegative).

Rabbits allotted into two equal groups; Control (non infected) group and infected group in which rabbits are experimentally inoculated with rabbit hemorrhagic disease virus (RHDV) through nostril by $1 \mathrm{ml}$ of liver haemogenate containing RHDV with Haemagglutination titer 1:1280, a local virulent field strain was isolated and identified by (Metwally and Madbouly, 2005). All rabbits were dissected after 24, 48 and 72 hrs post infection. All rabbits were subject to the full local protocols and scientific research ethics on animals as well as the application of security requirements and bio-security through all the steps of experiment.

\section{2-Clinical Symptoms and Post-Mortem Lesions:}

After experimental infection with RHDV, symptoms and post mortem lesions (PM) were recorded. Liver were collected and used for detecting the RHDV antigen by HA test and small intestine were collected for histopathological, histochemical

and immunohistochemical studies.

\section{3- Histopathological Examination:}

Both control and infected pieces of $0.5-1 \mathrm{~cm}$ in diameter of small intestine were fixed in $10 \%$ neutral buffered formalin solution, dehydrated in an ethanolic series, cleared in xylene and embedded in paraffin wax. $5 \mu$ paraffin sections were stained with haematoxylin and eosin method (H\&E) (Bancroft et al. 1996) and mounted in canda balsam. Slides were microscpically examined for photomicrographed.

\section{4- Histochemical Investigation:}

Alcian blue method for demonstrations of acid mucopolysaccharides was done (Steedman, 1950; Lison, 1954).

\section{5-Immunohistochemical Studies (Indirect Immunoperoxidase Test (IP):}

Small intestine sections were stained by indirect Immunoperoxidase test (Alonso et al. 1998). The sections were dewaxed and dehydrated, and endogenous peroxidase was blocked by $\mathrm{H}_{2} \mathrm{O}_{2} 0 \cdot 3 \%$ in methanol for 10 min. The sections were incubated with $10 \%$ normal rabbit serum as blocking normal serum in addition to biotin to avoid non specific staining sites. The sections were 
incubated with polyclonal Anti rabbit RHDV antibody (diluted in buffer 1: 20). This was followed by washing in three cycles of phosphate buffer saline (PBS) for $30 \mathrm{~min}$ each, and then incubates sections for 30 min with diluted goat anti-rabbit IgG peroxidase conjugate. After three further 15 min washes in PBS, a 5-min incubation in $0 \cdot 05 \%$ DAB containing $\mathrm{H}_{2} \mathrm{O}_{2} 0 \cdot 05 \%$ was used to identify peroxidase activity. The sections were counterstained with haematoxylin.

\section{6-Biochemical analysis:}

\subsection{1- Determination of serum aminotransferases} activity (AST, ALT):

The activity of aspartate aminotransferase (AST) and alanine aminotransferase (ALT) were estimated according to colorimetric method of Reitman and Frankel, (1957) using commercial kits "RANDOXS diagnostics AST/ALT kit, France.

\subsection{2- Determination of serum total protein:}

Serum total proteins determined according to the colorimetric method (Henry, 1964) using commercial kits "DIALAB diagnostics protein kit, Wien".

\subsection{3- Determination of serum albumin:}

Serum albumin determined by colorimetric method (Doumas and Biggs, 1972) by using commercial kits "ELITECH diagnostics albumine kit, France".

\subsection{4- Determination of serum creatinine:}

Serum creatinine determined by colorimetric method (Schirmeister, 1964) using commercial kits "DIALAB diagnostics creatinine kit, Wien".

\subsection{5- Determination of serum urea:}

Serum urea determined by colorimetric method (Friedman and Young, 1997) using commercial kits "BioSystems diagnostics urea kit, Spain".

\section{7- Statistical analysis:}

All data was statistical analyzed using SYSTAT program version 5 . The obtained data are shown in means \pm SE. where significant differences were calculated by multifactor analysis of variance (ANOVA) using Minitab (Minitab Inc. 2003).

\section{Results}

\section{1-Clinical symptoms and post mortem lesions:}

Clinical symptoms can be observed on infected rabbits as anorexia, depression, nervous signs (convolusion, ataxia, paddling), respiratory signs (dyspnea, frothy and bloody nasal discharge), cyanosis of mucous membranes and cries. (Figure 1a shows normal appearance of different visceral organs of control (non infected) rabbits. Both of lung, liver, small intestine, stomach, kidney, spleen and urinary bladder were apparently exhibit characteristic normal size, color and activity. After infection with RHDV most organs show gross lesions at different intervals 24, 48 and 72 hrs of infection. At 24 hrs post infection showing oedematous, congested and mottled lungs, small intestine with haemorrhagic spots and blood vessels engorged with blood, enlarged liver with yellowish-brown color, degenerated and friable with a marked lobular pattern, Stomach with necrotic dark spots and engorged blood vessels (Fig. 1b). Small intestine of 48hrs post infection showing numerous haemorrhagic spots and swollen stomach with necrotic spots (Fig. 1c). Small intestine of $72 \mathrm{hrs}$ post infection showing severe collaese of haemorrhage patches with thickened wall of intestinal mucosa, markedly enlarged urinary bladder filled with bloody urine (Fig. 1d). Liver of infected rabbits were positive for haemagglutination activity (HA) that characteristic for RHDV.

\section{2-Histopathological examination:}

Figure 2a showed section of the small intestine of control (non infected) group that exhibit normal feature; outer serosa, muscularis externa, submucosa, cross sections of intestinal glands (crypts) in lamina propria, tall regularly arranged villi, strips of smooth muscle fibers; muscularis mucosa extended in-between the intestinal glands. High magnified part of the previous section showed tall regular villi exhibiting a columnar lining epithelium with strait borders and goblet cells, and core of lamina propria with diffuse lymphatic tissue and small blood vessels (Fig 2b). At 24 hpi small intestine section showing moderate disorganized and destructive villi, increase cellular infiltration in lamina propria, proliferation of intestinal gland, wide submucosa with dilated and congested blood vessels (Fig. 2 c). Small intestine of 48 hrs post infection exhibits noticeable shortening, fusion and blunting of disorganized villi, crypts hyperplasia, marked cellular infiltration and haemorrhage in both lamina propria and submucosa with dilated congested blood vessels (Fig. 2 d). At $72 \mathrm{hpi}$ small intestine showing severe destruction and atrophy of villi with loss of their height and lining epithelium, severe atrophy of crypts, vacuolization and destruction in stromal connective tissue in most layers (Fig. 2e).

\section{3- Histochemical Investigation:}

Figure 3a exhibited mucopolysaccharides of small intestine of control rabbits that have a positive affinity to alcian blue staining in goblet cells, brush borders of the villi and crypts. They acquired a homogenously dense blue colouration throughout the mucosal layer. Small intestine of 24 hrs post infection showing noticeable positively stained goblet cells, it shows increase activity of goblet cells as intensive coloration (Fig. 3b). At 48 hpi small intestine section showing an increase amount of mucopolysaccharides of the mucosa, a number of the positively stained goblet cells at the base of villi and crypts showing a strong alcian blue reaction (Fig. 3c). $72 \mathrm{~h}$ post infection showing reduction in goblet cells and mucopolysaccharides materials of mucosa (Fig. 3d). 

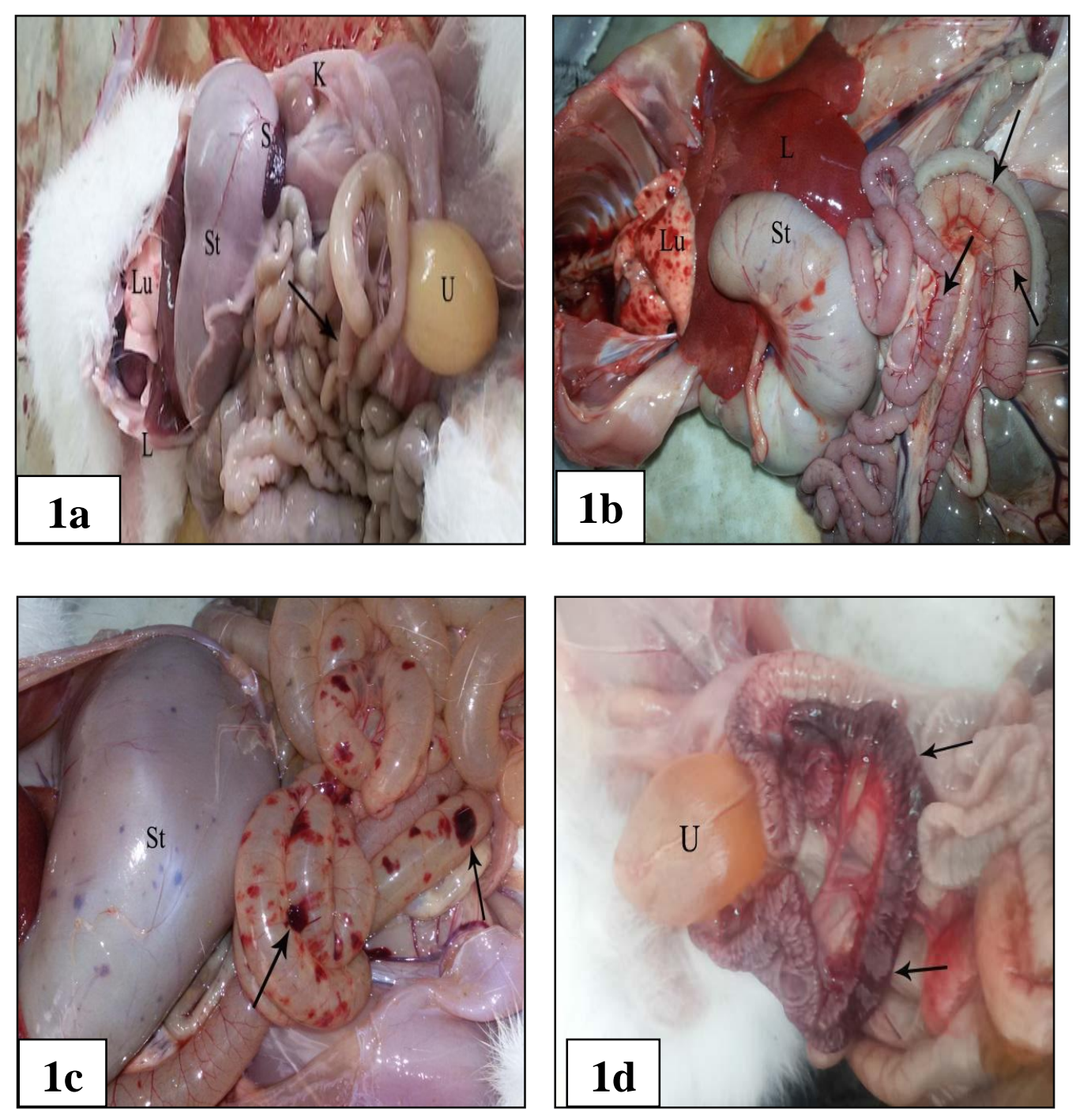

Figure 1a. visceral organs of control rabbits with normal appearance; lung (Lu), Liver (L), small intestine (arrow), stomach (St), kidney (K), spleen (S) and urinary bladder (U). Figs. 1 b-d. gross pathological lesions of infected rabbit organs include circulatory and degenerative disorders; some organs show slight to moderate swelling. Fig. 1b. 24 hrs after infection shows oedematous, congested and mottled lungs (Lu), small intestine with haemorrhagic spots and blood vessels engorged with blood (arrows), enlarged liver with yellowish-brown color, degenerated and friable with a marked lobular pattern (L), Stomach (St) with necrotic dark spots and engorged blood vessels. Fig. 1c. small intestine of 48 hrs post infected rabbits shows numerous haemorrhagic spots (arrows), swollen stomach with necrotic spots (St). Fig. 1d: 72 hrs post infected rabbits showing severe collaese of haemorrhage patches with thickened wall of intestinal mucosa (arrows), markedly enlarged urinary bladder filled with bloody urine (U). 

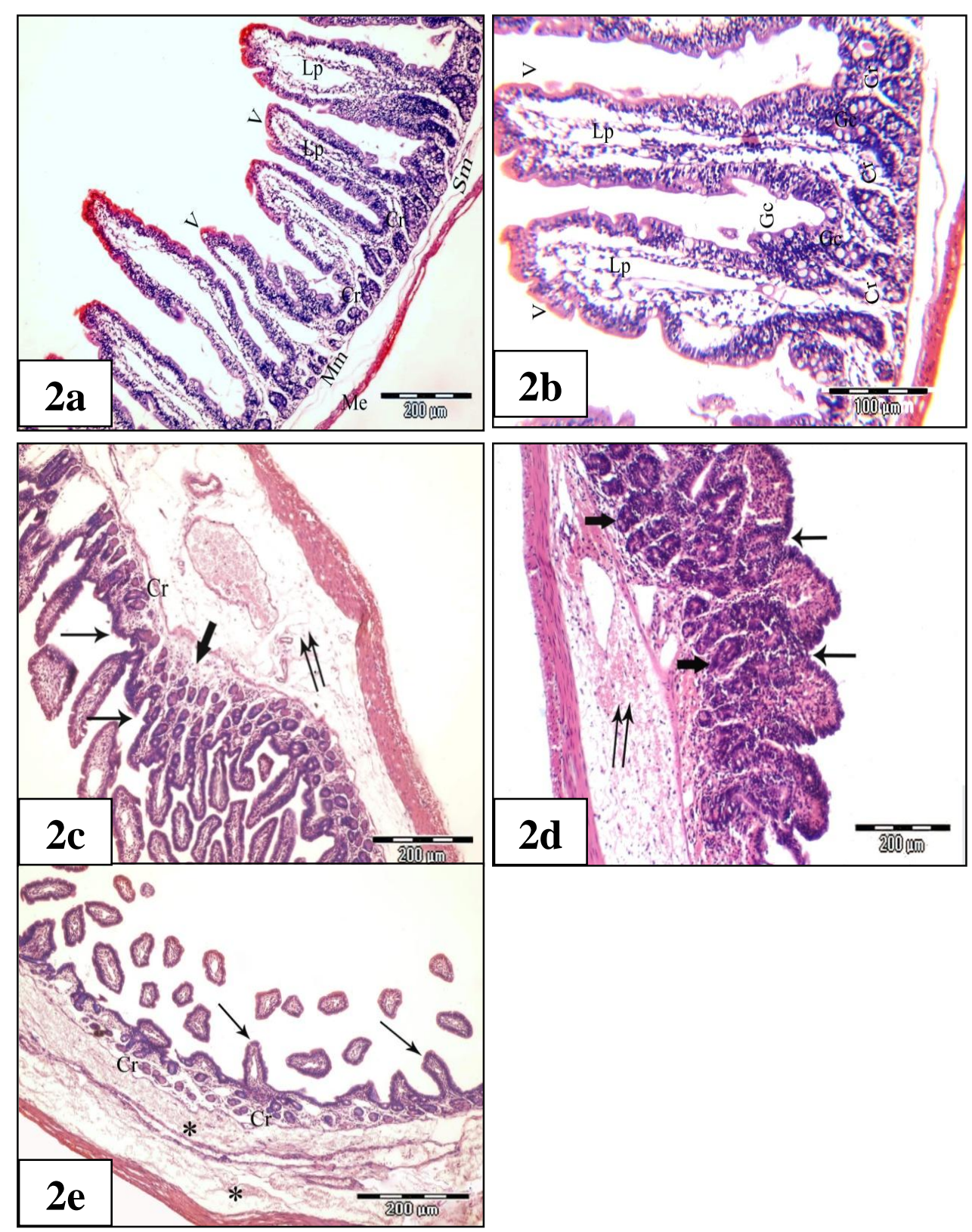

Figs. 2 a,b. light micrographs of histological sections of small intestine of control (non infected) animals stained with haematoxylin and eosin. Fig. 2a. transvere section of small intestine to show normal feature; outer serosa, Muscularis externa (Me), submucosa $(\mathrm{Sm})$, cross sections of intestinal glands (crypts)(Cr) in lamina propria (Lp), tall regularly arranged villi (V), strips of smooth muscle fibers; Muscularis mucosa (Mm) extended in-between the intestinal glands. Fig. 2b. high magnified part of the previous section shows tall regular villi (V) exhibiting a columnar lining epithelium with strait border and goblet cells (Gc), and core of lamina propria (Lp) with diffuse lymphatic tissue and small blood vessels. Figs. 2 c,d,e. light micrographs of small intestine sections of infected rabbits stained by haematoxylin and eosin showing disorganization and destructive features in particular mucosa and submucosa layers.

Fig. 2c. transvere section of small intestine 24 hrs post infection shows moderate disorganized and destructive villi (arrows), increase cellular infiltration in lamina propria (thick arrow), proliferation of intestinal gland (Cr), wide submucosa with dilated and congested blood vessels (two arrows). Fig. 2d. transvere section of 48 hrs post infection showing noticeable shortening, fusion and blunting of disorganized villi (arrows), crypts hyperplasia (thick arrow), marked cellular infiltration and haemorrhage in both lamina propria and submucosa with dilated congested blood vessels (two arrows). Fig. 2e. transvere section of small intestine after 72 hrs of infection show severe destruction and atrophy of villi with loss of their height and lining epithelium (arrows), severe atrophy of crypts (Cr), vacuolization and destruction in stromal connective tissue in most layers (stars) 

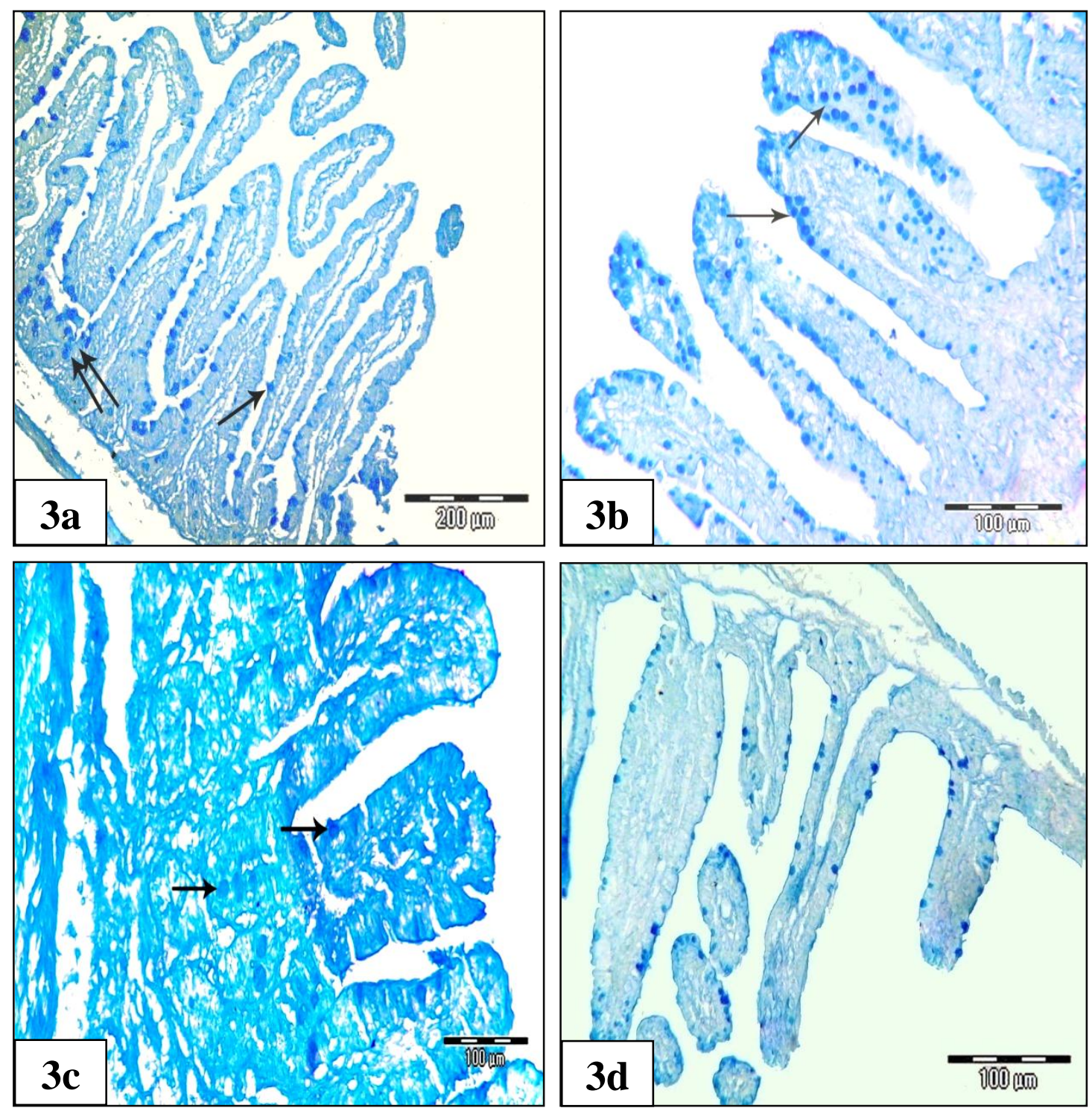

Figure 3 a-d. sections of small intestine of control and infected rabbits (Alcian blue preparations). Fig. 3a. section of small intestine of control rabbits showing positive affinity to the stain in goblet cells, brush borders of the villi (arrow) and crypts (two arrows). Fig 3b: Section of small intestine of 24 hrs post infection showing villi exhibit increase activity of goblet cells as intensive coloration (arrows). Fig 3c. section of small intestine of 48 hrs post infection showing an increase in acid mucopolysaccharides content of the mucosa, a number of the positively stained goblet cells at the base of villi as well as crypts exhibited a strong alcian blue reaction (arrows). Fig. 3d. at 72 hrs post infection showing reduction in goblet cells and mucopolysaccharides materials of mucosa. 

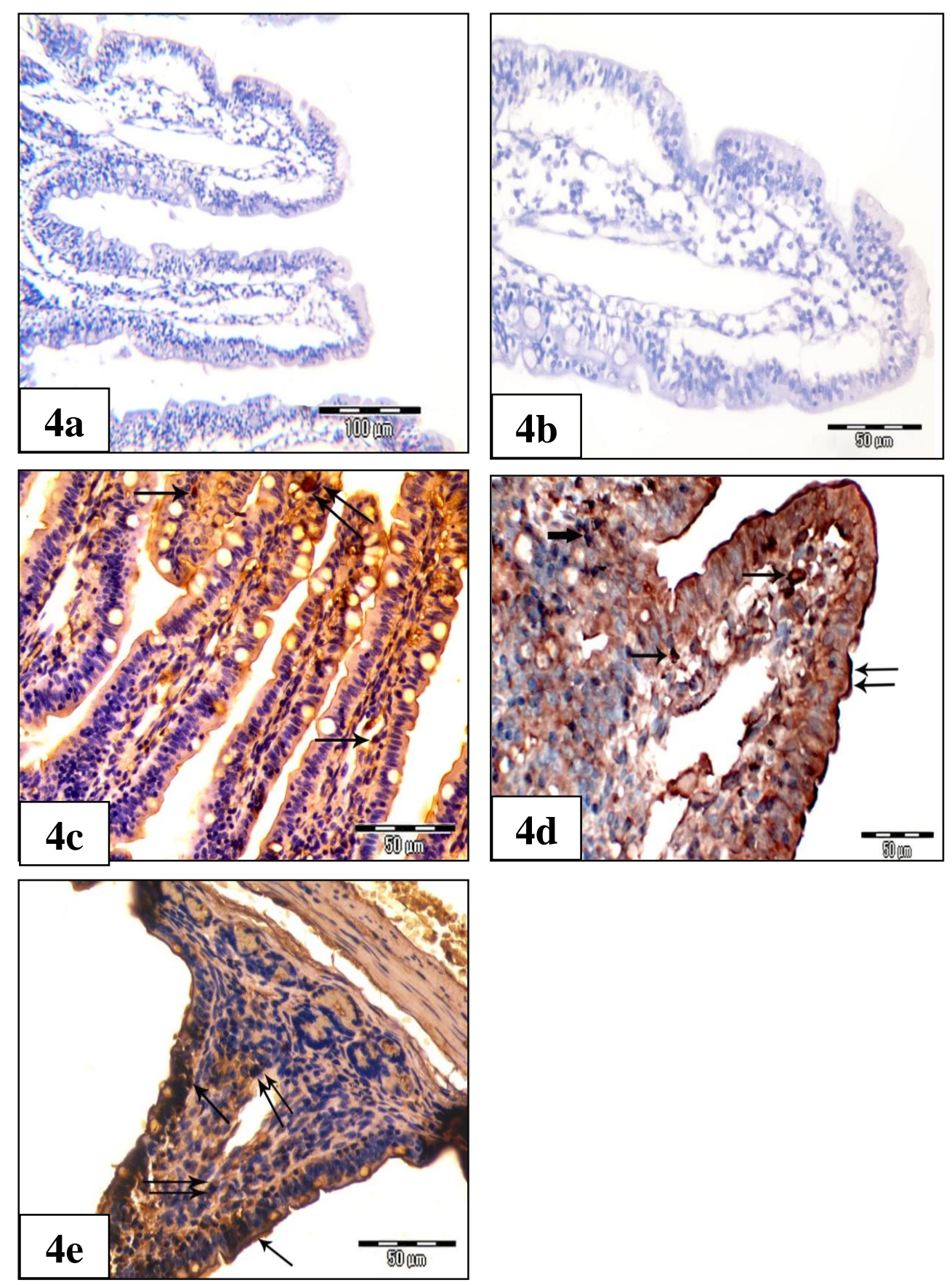

Figure 4 a-e. sections of small intestine stained with anti-RHDV immunostain and counterstained with haematoxylin in different experimental groups. Figs $\mathbf{4}$ a,b: low and high magnified sections of small intestine of control (non infected) rabbits showing negative reaction to anti- RHDV immunostain. Fig. 4c. section of 24 hrs post infection showing distinct cytoplasmic activity in macrophages (arrows) and presence of few number of apoptotic cells (two arrows). Fig. 4d. section of 48 hrs post infection showing highly positive reaction to antigen in the brush borders (two arrows), macrophages in the lamina propria (thick arrow) as well as moderate number of apoptotic cells can be observed (arrows). Fig. 4e. section of 72 hrs post infection showing strong cytoplasmic activity in the intraepithelial lymphocytes, brush borders of epithelial cells (arrows) and macrophages in the lamina propria (two arrows). 


\begin{abstract}
3.4- Immunohistochemical Studies Immunoperoxidase Test (IP):

Small intestine sections of control (non infected) rabbits immunostained for RHDV detection showed negative reaction to anti-RHDV immunostain (Fig. 4 a,b). At 24 hpi small intestine showing positive reaction restricted to the upper villi, minute cellular aggregation of lymphocytes, macrophages and neutrophils in the lamina propria, distinct cytoplasmic activity in macrophages and presence of few number of apoptotic cells. Fig. 4c exhibits highly positive reaction to antigen in the brush borders, macrophages in the lamina propria as well as moderate number of apoptotic cells can be observed after $48 \mathrm{hrs}$ pi. In the late stage of infection showing many RHDV positive cells accompanied by apoptosis, strong cytoplasmic activity in the intraepithelial lymphocytes, brush borders of epithelial cells and macrophages in the lamina propria (Fig. 4d).
\end{abstract}

\section{5- Biochemical analysis:}

Biochemical analysis of serum aspartate amino transferase (AST) and alanine amino transferase (ALT) indicated high significant increase of infected groups $(p \leq 0.001)$ comparing with control group. The effect of RHDV infection on serum albumin level showed no significant difference in both 24 and 48 hrs post infection but at 72 hrs of infection there was moderate significant decrease compared with control group $(p \leq 0.01)$ as well as infected rabbits with RHDV induced moderate significant increase in serum total protein of both 24, 48 hrs of post infected rabbits $(\mathrm{p} \leq 0.01)$ but no significant difference in 24 hrs post infected group compared with control group. Moreover, urea and creatinine level in serum induced high significant increase $(\mathrm{p} \leq 0.001)$ especially after $72 \mathrm{hrs}$ (Table1).

\section{Discussion}

Rabbit haemorrhagic disease (RHD) is a highly contagious devastating disease with dramatic economic losses caused by a calicivirus. The virus primarily causes infection of the liver, small intestine and spleen, sudden death is common and is usually due to a severe acute coagulopathy, probably instigated by the presence of virus on red blood cells. Congestion, haemorrhages and infarction of many organs are terminal events caused by the coagulopathy, but are not pathognomonic signs of RHD. In the present histopathological findings observed in the small intestine tissue of RHDV infected rabbits are a wide spread haemorrhage as a result of vascular damage, degeneration and necrosis. The most findings in the small intestine sections are erosions and disorganization of epithelial cells and the mucosal layer lost its integrity, some villi become abroad and adjacent to each other, vascular congestion, haemorrhage as well as proliferation and fusion of crypts can be seen. At 72 hrs post infection small intestine showing severe haemorrhage, necrosis of many epithelial cells, severe atrophy, destruction of villi and marked loss of its height. This agree with Gregg and House, (1989) who indicated that small intestine lesion is a characteristic features of RHD including necrosis of the small intestinal crypts, subsequent villous atrophy and sloughing of infected villous epithelial cells into the intestinal lumen, This may vary in severity and is often a segmental lesion involving only a few areas of the small intestine at a time.

In the present study, the clinical signs and post mortem lesions of experimentally infected rabbits also reisolation of RHDV from infected liver as well as histopathological findings, furthermore detection of RHDV particles within intestinal layers by immunohistochemical technique confirm the association of RHDV with macroscopic and microscopic lesions of intestine.

In the present study, small intestine section of control non infected rabbits immunostained for RHDV detection showing negative reaction to anti-RHDV immunostain. At 24 hpi small intestine showing positive reaction restricted to the villi tips, minute cellular aggregation of lymphocytes, macrophages and neutrophils in the lamina propria, distinct cytoplasmic activity in macrophages and presence of few number of apoptotic cells. At 48 hpi exhibits highly positive reaction to antigen in the brush borders, macrophages in the lamina propria as well as moderate number of apoptotic cells can be observed. In the late stage of infection showing many RHDV positive cells accompanied by apoptosis, strong cytoplasmic activity in the intraepithelial lymphocytes, brush borders of epithelial cells and macrophages in the lamina propria. On the same regard previous authors (Kevin et al. 2012) recorded that Immunohistochemical analysis of small intestine from the subcutaneously inoculated kit that died 48 hrs post infection show areas of focal necrosis and epithelial cells showing strong immunolabeling in the intestinal villi.

Localization of viral antigens by immunohistochemistry revealed that cells with apoptotic morphology were frequently expressing viral antigens as long as they retained their cytoplasmic structure. All cell types supporting viral replication presented apoptosis upon infection. Those cell types included macrophages, intravascular monocytes, and endothelial cells (StoerckleBerger et al. 1992).

Serum biochemistry revealed highly significant increase in AST, ALT, urea and creatinin but there were no significant difference in the level of albumin and slight significant difference in the level of total protein. Similary, Sheng et al. (2011) suggested that exponential increases in AST and ALT would be a strong prediction 
Table 1: Effect of RHDV infection on serum (AST), (ALT), Albumin, Total protein, Urea and creatinine in control and infected groups after 24, 48 and 72 hrs of infection.

\begin{tabular}{|c|c|c|c|c|c|c|c|}
\hline & AST & ALT & Alb & T.Prot & Urea & Creatinine \\
\hline \multicolumn{2}{|c|}{$\begin{array}{c}\text { Control } \\
\text { group }\end{array}$} & $7 \pm 0.57^{d}$ & $12 \pm 0.28^{d}$ & $3.82 \pm 0.01^{a}$ & $5.6 \pm 0.11^{c}$ & $32.42 \pm 1.39^{c}$ & $0.67 \pm 0.03^{b}$ \\
\hline \multirow{3}{*}{ 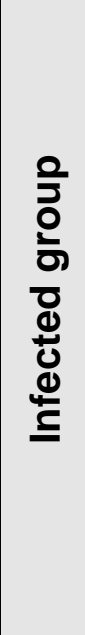 } & 放 & $27 \pm 1.15^{\mathrm{c}^{\star \star *}}$ & $17 \pm 0.86^{\mathrm{c}^{\star \star}}$ & $3.8 \pm 0.11^{\mathrm{a}}$ & $6 \pm 0.28^{b, c}$ & $48 \pm 1.15^{b^{\star \star}}$ & $1.6 \pm 0.34^{\mathrm{a}^{* *}}$ \\
\hline & 党 & $47 \pm 3.46^{b^{\star \star \star}}$ & $22 \pm 0.57^{b^{\star \star *}}$ & $3.9 \pm 0.12^{a}$ & $6.72 \pm 0.1^{\mathrm{a}^{\star \star \star}}$ & $45.5 \pm 6.06^{b^{\star \star *}}$ & $1.65 \pm 0.19^{\mathrm{a}^{\star \star}}$ \\
\hline & ज़ & $134 \pm 2.31^{\mathrm{a}^{* \star *}}$ & $62 \pm 1.15^{a^{\star \star \star}}$ & $3.4 \pm 0.01^{b^{\star *}}$ & $6.43 \pm 0.07^{a, b^{\star k *}}$ & $100 \pm 1.73^{a^{\star \star \star *}}$ & $2.2 \pm 0.12^{a^{\star \star \star}}$ \\
\hline
\end{tabular}

$*=$ low significance at $(\mathrm{p} \leq 0.05),{ }^{* *}=$ Moderate significance at $(\mathrm{p} \leq 0.01)$

$* * *=$ high significance at $(\mathrm{p} \leq 0.001), \mathrm{n}=9$ animals for each group

-Means in the same column with different small letter are significant difference.

of the fulminant consequence in RHDV infection and the profound changes in serum liver enzymes, particularly AST and ALT, may be used as useful parameters in monitoring the progression of RHDV infection.

It could be concluded that RHDV associated with induction of macroscopic and microscopic intestinal lesions along the course of disease. According to the available literature, this is the first report on association of RHDV with intestinal lesions. Further studies are required to clarify more about this infection in rabbits.

\section{References}

Alonso, C., Oviedo, J. M., Martin-Alonso, J. M., Diaz, E., Boga, J. A. and Parra, F. (1998): Programmed cell death in the pathogenesis of rabbit hemorrhagic disease. Archives of Virology 143, 321-332.
Bancroft, J. D., Steven, A. and Turner, D. R. (1996): Theory and practice of histological techniques. $4^{\text {th }}$ ed., Churchill living stone Edinburgh. London, Melbourne and New York.

Doumas, B. T. and Biggs, H. G. (1972): Determination of serum albumin Standard Methods of Clinical Chemistry. Acad. Press N. Y. 7: 175.

Friedman and Young (1997): Effects of disease on clinical laboratory tests, 3th ed. AACC press, 1997.

Gregg, D. A. and House C. (1989): Necrotic hepatitis of rabbits in Mexico. a Parvovirus. Vet. Rec., 125, 603-604.

Gregg, D. A., House, C. and Berninger, M. (1991): Viral haemorrhagic disease of rabbits in Mexico: epidemiology and viral characterization. Rev. Sci. tech. Off. int. Epiz., 1991, 10 (2),435-451

Henry, R. J. (1964): Clinical Chemistry, Harper and Row Publishers, New York,P. 181. 
Kevin P. Dalton, Inés Nicieza, Ana Balseiro, María A. Muguerza, Joan M. Rosell, Rosa Casais, Ángel L. Álvarez, and Francisco Parra (2012): Variant Rabbit Hemorrhagic Disease Virus in Young Rabbits, Spain. Emerging Infectious Diseases• www.cdc.gov/eid, Vol. 18, No. 12, December 2012

Liu, S. J., Xue, H. P., Pu, B. Q. and Qian, N.H. (1984): A new viral disease in rabbits. Animal Husbandry and Veterinary Medicine, (in Chinese). 16 (6): 253-255

Lison, M., (1954): Animal tissue technique $3^{\text {rd }}$ edition. San Francisco: W. H. Freeman company.

Metwally, A. Y. and Madbouly, H. M. (2005): Study on a new isolateof rabbit haemorrhagic disease virus. Bani-Suef Vet. Med. J., 15 (2): 283-243.

Meyers, G., Wirblich, C. and Thiel, H. J. (1991a): Rabbit hemorrhagic disease virus-molecular cloning and nucleotide sequencing of a calicivirus genome. Virology 184: 664-676

Meyers, G., Wirblich C. and Thiel H. J. (1991b): Genomic and subgenomic RNAs of rabbit hemorrhagic disease virus are both protein linked and packaged into particles. Virology 184: 677-686

Minitab Inc., (2003). Minitab Statistical Soft ware, Release 14 for Windows State College. PA

Parra, F. and Prieto, M. (1990): Purification and characterization of a calicivirus as the causative agent of a lethal hemorrhagic disease in rabbits. J. V. 64: 4013-4015
Reitman, S. and Frankel, S. (1957): A colorimetric method for the determination of serum glutamic oxalacetic and glutamic pyruvic transaminases. Am. J. Clin. Pathol. 28: 56- 63.

Schirmeister, J. (1964): Determination of creatinine in serum. Dtsch Med Wschr. 89: 1940.

Sheng, Y. C., Chi. C. C., Ming, T. L., Jui. H. S. and Chau. L. T. (2011): Exponential Increases in Serum Liver Enzymes in Fulminant Rabbit Haemorrhagic disease virus. J. Anim. Diagn. Med. (2011) 1: 13-19

Steedman, H. F. (1950): Alcian blue 8GX: A new stain for mucin. Quart. J. Micr. Sci. 91: 477-479

Stoerckle-Berger, N., Keller-Berger, B., Ackerman, M. and Ehrensperger, F. (1992): Immunohistological diagnosis of rabbit haemorrhagic disease (RHD). J. Vet. Med. 39: 237-245.

Ueda, K., Park, J. H., Ochiae, K. and Itakura, C. (1992): Disseminated intravascular coagulation (DIC) in rabbit haemorrhagic disease. Jpn. J. Vet. Res. 40: 133141.

World Organisation for Animal Health (2015). Chapter 13. 2.Rabbit haemorrhagic disease. In Terrestrial Animal Health Code, Twentyfourth edition. OIE, Paris 